\title{
EL FINANCIAMIENTO Y SU INFLUENCIA EN LOS INGRESOS POR VENTAS: CASO EMPRESA LECHE GLORIA S.A. 1998-2018
}

\author{
FINANCING AND ITS INFLUENCE ON SALES REVENUE: LECHE GLORIA S.A. \\ CASE 1998-2018
}

\section{RESUMEN}

Objetivo: Analizar el nivel de influencia del financiamiento en el crecimiento de ingresos por ventas de la empresa Leche Gloria S.A. (Ex Gloria S.A.) durante el periodo 1998-2018. Método: El enfoque de la investigación es cuantitativo, el tipo de investigación es básica con un nivel descriptivo, correlacional-explicativo, en un diseño no experimental y longitudinal; es decir, se realiza la investigación con información documental reportada a la Superintendencia del Mercado de Valores (SMV) y a la Bolsa de Valores de Lima (BVL). Resultados: El patrimonio se incrementó a una tasa de $6,72 \%$ promedio anual (56.36 millones promedio por año), la deuda financiera aumentó a una tasa de $5,87 \%$ promedio anual (29.75 millones promedio por año) y los ingresos por ventas se incrementaron en $9,09 \%$ promedio anual (144.4 millones promedio por año). Conclusiones: Se confirma que el financiamiento con deuda financiera y con patrimonio influyeron positiva y significativamente en los ingresos por ventas de la empresa Leche Gloria S.A. durante el periodo 1998-2018. Se demostró de manera individual mediante el estadístico t de Student, $\mathrm{t}_{\mathrm{c}-\mathrm{Pat}}=17,20 \mathrm{y} \mathrm{t}_{\mathrm{c}-\mathrm{DF}}=2,64$ mayores que $t_{t}=2,10$ y del modelo con el estadístico $F, F_{c}=440,00$ mayor que $\mathrm{F}_{\mathrm{t}}=3,55$.

Palabras clave: Deuda financiera; ingresos; ventas; patrimonio; empresa.

\begin{abstract}
Objective: To analyze the level of influence of financing in the sales revenue growth in Leche Gloria S.A (the former Gloria S.A.). company during the period 1998-2018. Method: The research approach is quantitative, the type research is basic with a descriptive, correlate - explanatory level in a non-experimental, longitudinal design, it means, perform the investigation with documentary information reported to the Superintendence of the Securities Market (SMV). Results: the equity increased in a rate of $6.72 \%$ annual average (56.36 million average per year), the financial debt increased in a rate of $5.87 \%$ annual average $(29.75$ million average per year) and the sales revenue increased $9.09 \%$ annual average (144.4 million average per year). Conclusions: It is confirmed that financing with financial debt and with equity influenced positive and significantly the sales revenue in Leche Gloria S.A. company during the period 1998-2018, has been shown in a individual way through the statistic $t$ of Student , tc-Pat $=17,20$ and $t c-D F=2,64$ greater than $t t=2,10$ and the model with the $F$ statistic, $\mathrm{Fc}=440,00$ greater than $\mathrm{Ft}=3,55$.
\end{abstract}

Keywords: Financial debt; income; sales; equity; company.

(c) Los autores. Este artículo es publicado por la Revista Quipukamayoc, Universidad Nacional Mayor de San Marcos. Este es un artículo de acceso abierto, distribuido bajo los términos de la Licencia Creative Commons Atribución-NoComercial-Compartirlgual 4.0 Internacional.(http://creativecommons.org/licenses/by-nc-sa/4.0/), que permite el uso no comercial, distribución y reproducción en cualquier medio, siempre que la obra original sea debidamente citadas. 


\section{INTRODUCCIÓN}

El Perú está pasando por más de 25 años de estabilidad económica, factor importante para el crecimiento económico del país y de sus empresas. Al respecto, en el Reporte de Estabilidad Financiera de noviembre de 2018, el Banco Central de Reserva del Perú expresa que "la estabilidad financiera y la estabilidad monetaria son condiciones necesarias para el crecimiento sostenido de la economía" (p. 5). Asimismo, Vásquez y Zamudio (2013) afirman que "en los últimos años, el Perú ha registrado un significativo crecimiento económico descentralizado, impulsado principalmente por la dinámica del mercado interno" (p. 42).

El Producto Bruto Interno (PBI) real del Perú durante el periodo de estudio $1998-2018$ creció en 4,71\% promedio anual, impulsado por la demanda interna que creció en $4,66 \%$ promedio anual y por las exportaciones netas que crecieron en $6,59 \%$ promedio anual, siendo los factores macroeconómicos importantes en el crecimiento del sector empresarial y en particular de la empresa Leche Gloria S.A.

Bajo este contexto de crecimiento, las empresas requieren de mayor financiamiento para sus inversiones en capital de trabajo y en activos fijos y, de esa manera, coadyuvar a los ingresos por ventas. El financiamiento se busca en los mercados financieros nacionales e internacionales. Respecto a los mercados financieros, Madura (2008) manifiesta que son los que "facilitan el flujo de fondos con el fin de financiar las inversiones de las empresas, los gobiernos y los individuos" (p. 20).

Durante el periodo de estudio, la empresa corporativa Leche Gloria S.A. obtuvo financiamiento para sus inversiones, a través de préstamos bancarios y operaciones de leasing o arrendamiento financiero con diversas instituciones financieras nacionales y extranjeras, entre las principales tenemos: "Banco de Crédito del Perú, Scotiabank, BBVA Continental, Interbank, Interamericano de Finanzas, Citibank, PNC Bank, Commerce Bank, BNP Paribas sucursal Panamá, Santander, I.F.C. (Corporación Financiera Internacional) y HSBC Bank Perú S.A., actualmente Banco GNB Perú" (Bolsa de Valores de Lima, 2008, p.29).

Asimismo, la Superintendencia del Mercado de Valores (SMV) (ex CONASEV) durante el periodo de estudio otorgó autorizaciones a la empresa Leche Gloria S.A. para emitir bonos corporativos y papeles comerciales (deuda financiera) en el mercado de capitales peruano. La primera autorización se emitió dos años antes del periodo de estudio, mediante Resolución N. ${ }^{\circ}$ 218-96-EF/94.10 del 21 de mayo de 1996, por la suma de hasta 100 millones de soles; la segunda autorización fue dada mediante la Resolución N. . ${ }^{0}$ 11-2000 EF/94.11 del 26 de enero de 2000, por la suma de hasta US\$ 60 millones; la tercera autorización provino de la Resolución N. ${ }^{\circ}$ 041-2003-EF/94.11 del 13 de junio de 2003, por la suma de hasta US\$ 60 millones; la cuarta autorización derivó de la Resolución Gerencia General N.o 120-2006-F/94.11 de fecha 3 de noviembre de 2006, hasta por US\$ 120 millones; la quinta autorización resultó de la Resolución de Directoral de Emisores N. ${ }^{\circ}$ 122-2008-EF/94.06.3 de fecha 21 de agosto de 2008, hasta por US\$ 200 millones; y por último, la sexta autorización es la Resolución de Intendencia General N. ${ }^{\circ}$ 0642014-SMV/11.1 de fecha 22 de julio de 2014 por la suma de hasta US\$ 250 millones. La emisión de bonos corporativos (deuda financiera de largo plazo) fue utilizada para financiar las operaciones y las inversiones de la empresa (incluyendo la posibilidad de financiar pasivos).

Con la finalidad de sostener su crecimiento, posicionamiento y expansión en el mercado nacional e internacional, la empresa Leche Gloria S.A: ha requerido de financiamiento del mercado financiero nacional e internacional, del mercado de bancario y del mercado capitales como se señaló anteriormente. Al respecto Ross, Westerfield y Jaffe (2008) manifiestan que "como es evidente, el financiamiento externo necesario y el crecimiento están relacionados. Si no intervienen otros factores, cuanto más alta sea la tasa de crecimiento de ventas o activos, mayor será la necesidad de financiamiento externo" (p. 68).

Además, la empresa ha recurrido a un mayor financiamiento mediante la reinversión de un porcentaje de las utilidades netas del ejercicio y que van a formar parte de los resultados acumulados y a la vez del patrimonio de la empresa. Van Horne y Wachowicz (2010) manifiestan sobre el patrimonio inicial:

Financiamiento inicial. Cuando se constituye una compañía, es evidente que debe financiarse con frecuencia, el financiamiento inicial que proviene de los fundadores y sus familiares y amigos. En el caso de algunas compañías, esto es suficiente para la puesta en marcha, y si retienen utilidades en el futuro ya no necesitarán financiamiento de capital accionario externo. Para otras son necesarias las infusiones de capital accionario externo adicional. (p. 519)

El financiamiento está representado por lo que se llama estructura financiera y estructura de capital. Para este trabajo de investigación se ha utilizado la estructura de capital o la capitalización de la empresa, que según Weston y Copeland (1992) "es el financiamiento permanente representado por la deuda a largo plazo, por las acciones preferentes y por el capital de los accionistas [...] La estructura financiera se refiere a la forma en que son financiados los activos de la empresa" (p. 579). El financiamiento con deuda financiera y patrimonio de los activos (inversiones) respalda los ingresos por ventas de la empresa. 
Se completa el fundamento de financiamiento sobre los ingresos por ventas en base a lo que Moyer, McGuigan y Kretlow (2005) expresan:

Así, para mantener las estructuras deseadas de capital, las empresas tienen que aumentar de manera periódica la deuda a largo plazo. Este refinanciamiento gradual de la deuda, junto con la deducción fiscal de los intereses, explica el hecho de que aproximadamente entre el $85 \%$ y 90\% del capital externo a largo plazo que se obtiene en Estados Unidos sea de forma de deuda. (p. 209)

Con respecto a los ingresos por ventas y a su crecimiento, Bravo (2017) expresa:

Es tal vez importante tomar decisiones de financiamiento para facilitar el crecimiento de la empresa a través de deuda o emisión de acciones. Siempre será importante saber si la empresa es o no rentable para sus accionistas, si genera valor, si se incrementa el patrimonio de los accionistas. Si la empresa está creciendo será tal vez la oportunidad de invertir en una expansión agresiva o ver la posibilidad de comprar negocios complementarios. (párr. 1)

Para la revista Forbes México (2016) existen siete pilares para el crecimiento de una empresa. Uno de los pilares es el financiamiento y finanzas: "Todos los negocios necesitan fondos para crecer. Las empresas líderes determinan la mejor solución financiera - o mezcla de soluciones - para sus negocios y obtener beneficios máximos de su gestión de los fondos disponibles" (párr. 12).

En el sentido antes descrito, la hipótesis planteada es que el financiamiento influye en el crecimiento de ingresos por ventas en la empresa Leche Gloria S.A., también Levy (2017) manifiesta "nuestra hipótesis es que el dinero antecede la producción, aumenta tanto el ingreso como el ahorro y genera liquidez para realizar los bienes de capital fijo" (p. 209).

\section{MATERIALES Y MÉTODOS}

El enfoque de la investigación es cuantitativo, es decir, objetivo y sistemático. El tipo de investigación es básica con un nivel descriptivo, correlacional - explicativo en un diseño no experimental y longitudinal.

Con el objetivo de analizar el financiamiento y su influencia en los ingresos por ventas de la empresa Leche Gloria S.A., se revisaron los estados financieros que reporta a la Superintendencia del Mercado de Valores (SMV) y a la Bolsa de Valores de Lima (BVL) durante el periodo 19982018. Los datos fueron anuales y se obtuvieron del estado de situación financiera y del estado de resultados de la empresa en estudio.

La variable dependiente estuvo constituida por los ingresos por ventas de la empresa. Las variables independientes fueron: patrimonio neto (PA) y deuda financiera (DF).

Con el propósito de verificar la relación de las características del financiamiento de la empresa y de sus ingresos por ventas, se utilizó el análisis de regresión lineal múltiple, por el método de Mínimos Cuadrados Ordinarios (MCO) para la información obtenida durante 21 años, de 1998 a 2018.

A continuación, se presenta el modelo econométrico utilizado para analizar la influencia del financiamiento de las variables independientes deuda financiera (DF) y patrimonio $(\mathrm{PA})$ en la variable dependiente, ingreso por ventas $(\mathrm{V})$.

$$
V_{i}=\beta_{1}+\beta_{2} D F_{i}+\beta_{3} P A_{i}+u_{i}
$$

$\mathrm{B}_{1}$ : valor del intercepto

$\mathrm{B}_{2}, \mathrm{~B}_{3}$ : coeficientes de las variables explicativas

$\mu$ : presenta el término de error de la regresión.

Para obtener los resultados, los datos se procesaron utilizando el software estadístico EViews. Este es un software que compila e interpreta datos econométricos, generando los resultados para la regresión lineal múltiple por MCO.

\section{RESULTADOS}

Tal como se puede observar en la figura 1 los ingresos por ventas crecieron a una tasa de $9,06 \%$ compuesto anual durante el periodo de 1998 al 2016, obteniendo una participación importante en el mercado de alimentos; después, desde el 2017 al 2018, se aprecia un ligero decrecimiento causado por factores internos (riesgo operativo) y externos (crecimiento de la economía a 3,25\% promedio en el periodo analizado y crisis política).

Los datos organizados de las variables del modelo de investigación para el periodo 1998-2018 se presentan en la tabla 1.

Con respecto a la variable patrimonio, se deduce que el patrimonio de la empresa Leche Gloria S.A. durante el periodo 1998-2018 se incrementó en 1,127.22 millones de soles $(267,25 \%)$, que es equivalente a un crecimiento de $6,72 \%$ promedio anual (+56.36 millones de soles promedio por año) sustentado principalmente en la cuenta resultados acumulados que aumentó en 11,83\% promedio anual, los que a la vez se sustentan en la reinversión de 
utilidades netas del ejercicio que tuvieron un crecimiento promedio de 53.38 millones de soles promedio anual equivalente al $20,14 \%$ promedio anual.

Es importante señalar que en el año 2008, por motivos de reestructuración patrimonial, la empresa disminuyó su patrimonio en 76.89 millones de soles (- 7,5\% con respecto al año anterior) y en el año 2018 también disminuyó por política de la Junta General de Accionistas que decidió pagar dividendos en un monto de 238.7 millones de soles mayor a las utilidades del ejercicio de 160.706 millones de soles, disminuyendo el patrimonio en 104.62 millones con respecto al año anterior $(-6,3 \%)$ (Ver figura 1$)$.

Por su parte, la deuda financiera tuvo un crecimiento de 5,87\% promedio anual (+S/. 29.75 millones por año) durante el periodo de estudio (ver tabla 1 y tabla 2 ).

La deuda financiera tuvo incrementos altos, así como disminuciones también significativas durante el periodo de estudio (ver figura 3), esto debido a que la deuda mediante emisión de bonos se va cancelando periódicamente mediante el programa de amortización y luego se realizan nuevas emisiones de deuda, que la incrementan. Un ejemplo fue el que ocurrió a fines del año 2014 y durante el año 2015 , tiempo en el que se emitieron bonos corporativos por la suma de 593.02 millones de soles.

Los resultados estimados de la regresión lineal múltiple para la variable Ingresos por ventas se muestran en la tabla 2. Se observa que todos los coeficientes estimados son estadísticamente significativos porque la probabilidad de cada uno de ellos es menor de 0,05, asimismo, los t calculados son mayores que el $t$ de tabla de la distribución $t$ Student $\left(t_{t}=2,12\right)$.
De igual forma, la significatividad del modelo econométrico propuesto es alta, ya que el valor de $\mathrm{F}$ calculado de 440,00 es mayor que el valor F de tabla igual a 3,55 (probabilidad del valor de la prueba $\mathrm{F}$ es muy pequeño igual a 0,0000), como se observa en la tabla 3 .

Con los resultados obtenidos en la tabla 2, se presenta el modelo de los ingresos por ventas de la empresa leche Gloria S.A. que podría ser utilizado como instrumento para realizar su proyección de los ingresos por ventas de acuerdo con su planeación financiera.

Modelo 1:

$$
\mathrm{V}=-804,6679+2,2608^{\star} \mathrm{PAT}+0,8376 \mathrm{~F}
$$

El modelo 1 cumple con los supuestos de los MCO (ver tabla 3), no existe problemas de autocorrelación de primer orden, según el estadístico Durbin - Watson está cercano de 2,00, $(\mathrm{du}=1,538<\mathrm{dw}=1,83)$, cumple con el supuesto de normalidad de residuos medido con el estadístico Jarque Bera de 0,74; la prueba de heteroscedasticidad con el Test de White $\mathrm{pF}(5,15)=0,8876$, por tanto, se acepta el supuesto de homocedasticidad; y el diagnóstico de estabilidad con Chow Breakpoint Test pF $(3,15)=0,3713$, dado que la probabilidad es mayor a 0,05 , se acepta la estabilidad del modelo econométrico propuesto para este trabajo.

Decisión: visto los resultados se acepta la hipótesis propuesta, por lo tanto, se afirma que el financiamiento con patrimonio y con deuda financiera sí influyeron positiva y significativamente en los ingresos por ventas de la empresa Leche Gloria S.A. durante el periodo de estudio 1998-2018.

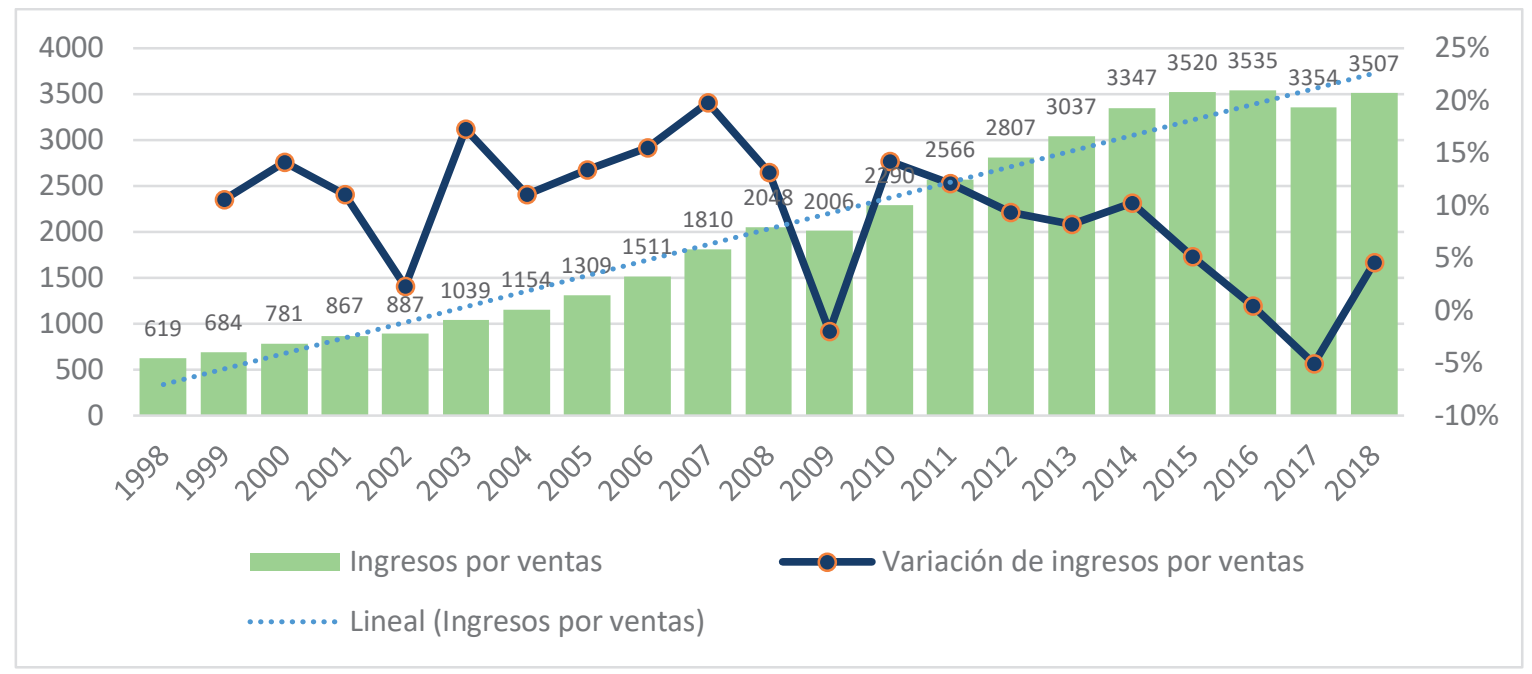

Figura 1. Evolución de Ingresos por ventas y su variación anual. Empresa Leche Gloria S.A. 1998-2018. Fuente. Elaboración propia en base a la tabla 1 
Tabla 1

Información de las variables independientes y dependiente (en millones de soles)

\begin{tabular}{|c|c|c|c|}
\hline Años & Ingresos por ventas & Deuda financiera & Patrimonio \\
\hline 1998 & 619,12 & 279,11 & 421,78 \\
\hline 1999 & 684,21 & 43887 & 483,41 \\
\hline 2000 & 780,91 & 424,71 & 527,70 \\
\hline 2001 & 867,03 & 388,60 & 574,24 \\
\hline 2002 & 886,66 & 397,76 & 650,05 \\
\hline 2003 & 1039,44 & 372,55 & 739,54 \\
\hline 2004 & 1154,28 & 350,34 & 786,45 \\
\hline 2005 & 1308,97 & 398,06 & 851,10 \\
\hline 2006 & 1511,45 & 358,62 & 945,50 \\
\hline 2007 & 1810,42 & 493,28 & 1019,18 \\
\hline 2008 & 2047,74 & 467,22 & 942,29 \\
\hline 2009 & 2006,17 & 475,45 & 1170,94 \\
\hline 2010 & 2290,38 & 406,69 & 1237,74 \\
\hline 2011 & 2566,41 & 447,58 & 1335,97 \\
\hline 2012 & 2806,69 & 44169 & 1399,52 \\
\hline 2013 & 3036,94 & 416,72 & 1450,48 \\
\hline 2014 & 3347,45 & 642,98 & 1543,86 \\
\hline 2015 & 3519,76 & 752,20 & 1601,00 \\
\hline 2016 & 3534,53 & 835,35 & 1596,56 \\
\hline 2017 & 3353,75 & 790,07 & 1653,61 \\
\hline 2018 & 3507,06 & 874,16 & 1548,99 \\
\hline Variación (\%) & $9,06 \%$ & $5,87 \%$ & $6,72 \%$ \\
\hline
\end{tabular}

Fuente. Bolsa de Valores de Lima, Superintendencia del Mercado de Valores / Elaboración propia.

Tabla 2

Regresión lineal múltiple de la variable dependiente Ingresos por ventas

\begin{tabular}{|c|c|c|c|c|}
\hline \multicolumn{3}{|c|}{ Dependent Variable: V } & R-squared & 0,980 \\
\hline \multicolumn{3}{|c|}{ Method: Least Squares } & Adjusted R-squared & 0,978 \\
\hline F-statistic & \multicolumn{2}{|l|}{440,00} & Durbin-Watson stat & 1,829 \\
\hline Prob(F-statistic) & \multicolumn{2}{|l|}{0,0000} & Date: 03/13/20 Time: 00:12 & \\
\hline \multicolumn{3}{|c|}{ Included observations: 21} & \multicolumn{2}{|l|}{ Sample: 19982018} \\
\hline Variable & Coeficiente & Std. Error & t-Statistic & Probabilidad \\
\hline C & $-804,67$ & 110,38 & $-7,29$ & 0,000000 \\
\hline PA & 2,26 & 0,13 & 17,20 & 0,000000 \\
\hline DF & 0,84 & 0,32 & 2,64 & 0,016700 \\
\hline
\end{tabular}

Fuente. Elaboración propia en base a los datos de la tabla 2. 


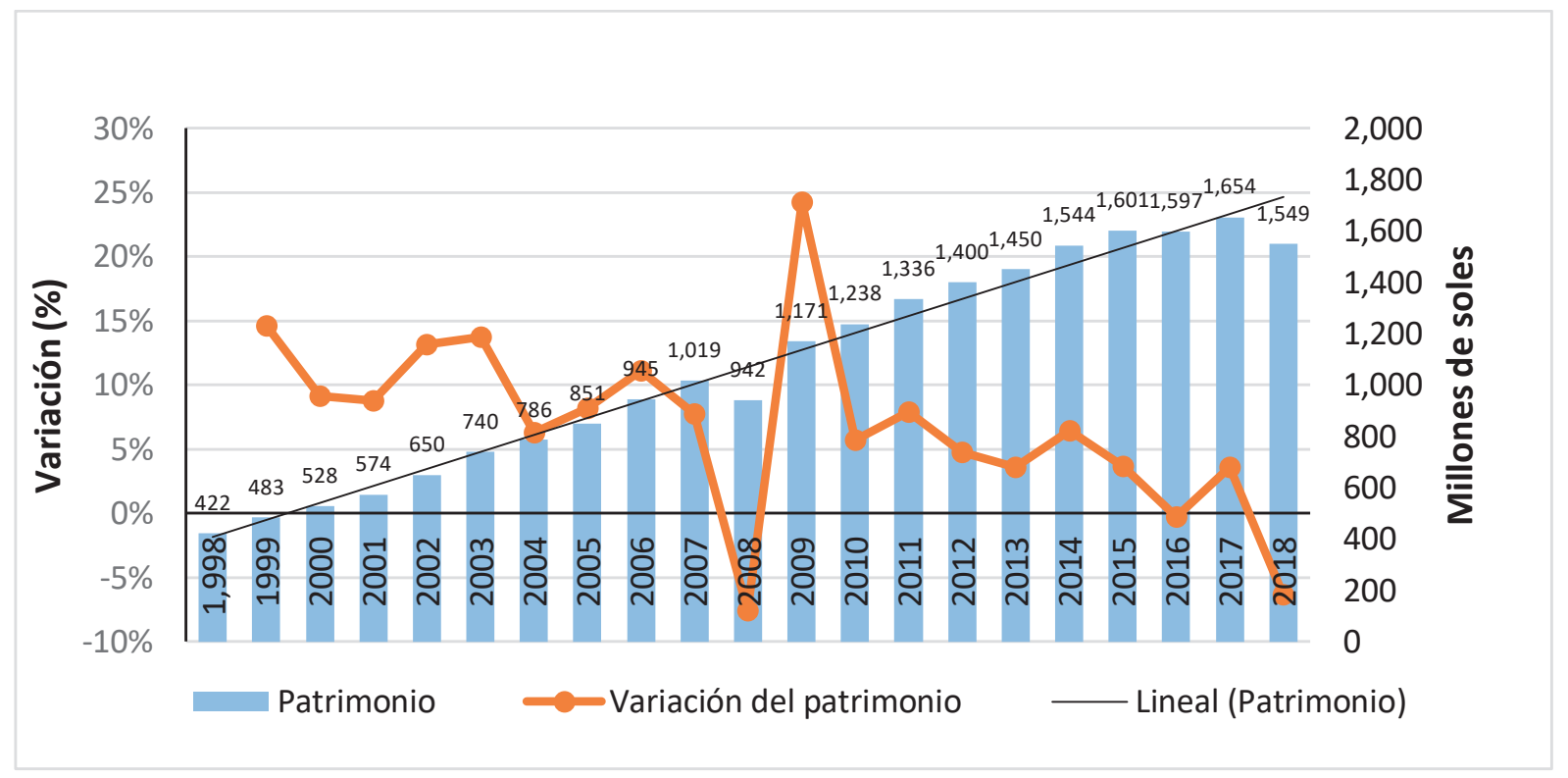

Figura 2. Evolución del Patrimonio y su variación anual. Empresa Leche Gloria S.A. 1998-2018. Fuente. Elaboración propia en base a la tabla 1

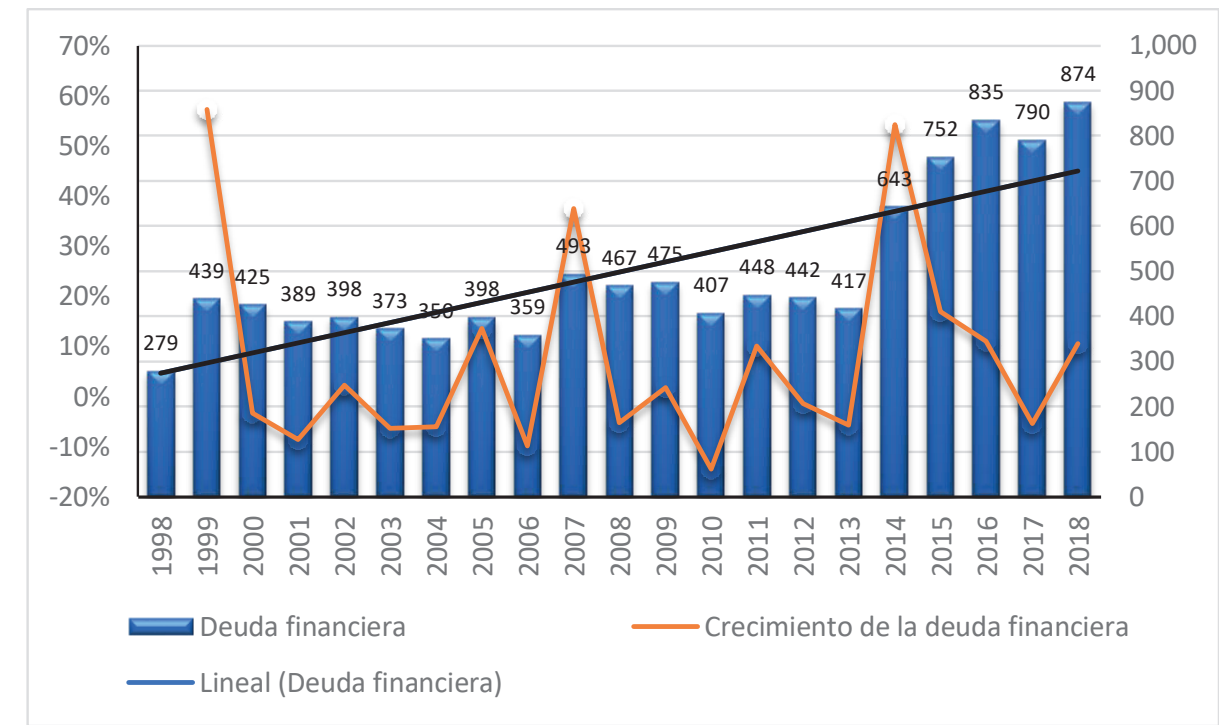

Figura 3. Evolución de deuda financiera y su variación anual. Empresa Leche Gloria S.A. 1998-2018. Fuente. Elaboración propia en base a la tabla 1

Tabla 3

Resultado de cumplimiento de los supuestos de MCO

\begin{tabular}{lcccc}
\hline Homocedasticidad & No Autocorrelación & No Multicolinealidad & Normalidad de residuos & Estabilidad \\
\hline Test White & Durbin Watson & Índice de condicionalidad & Jarque Bera & Chow Breakpoint Test: 2009 \\
Prob. F $(5,15)$ & Estadístico & Coeficiente & Estadístico & Prob. F (3,15) \\
0,8876 & 1,83 & $0,61 \mathrm{y}$ & 0,3713 \\
\hline
\end{tabular}

Fuente. Elaboración 
Considerando la regresión del modelo econométrico propuesto, podemos establecer que la contrastación de la hipótesis planteada indica que las dos variables del financiamiento influyen positivamente sobre los ingresos por ventas de la empresa Leche Gloria S.A.

Por consiguiente, se demostró estadísticamente que hay influencia positiva y significativa de la deuda financiera con los ingresos por ventas de la empresa Leche Gloria S.A. Como se observa en la figura 4, el diagrama de dispersión y el coeficiente de correlación de 0,65 corroboran que la relación entre deuda financiera e ingresos por ventas es positiva y directa, es decir, si aumenta el financiamiento con deuda financiera, los ingresos por ventas se incrementan positivamente.
Asimismo, se demostró estadísticamente que hay influencia positiva y significativa del patrimonio con los ingresos por ventas de la empresa Leche Gloria S.A. Como se observa en la figura 5, el diagrama de dispersión y el coeficiente de correlación de 0,9722 , evidenciando que la relación entre patrimonio e ingresos por ventas es positiva y directa, es decir, si aumenta el financiamiento con patrimonio, los ingresos por ventas se incrementan positivamente.

En resumen, como se observa en la tabla 2, el modelo econométrico, arroja un $\mathrm{R}^{2}$ y el $\mathrm{R}^{2}$ ajustado de 0,980 y 0,978 respectivamente, los cuales son muy altos, explicando las variables deuda financiera y patrimonio más del 97,8\% de la variabilidad de los ingresos por ventas de la empresa Leche Gloria S.A.; es decir, existe una buena

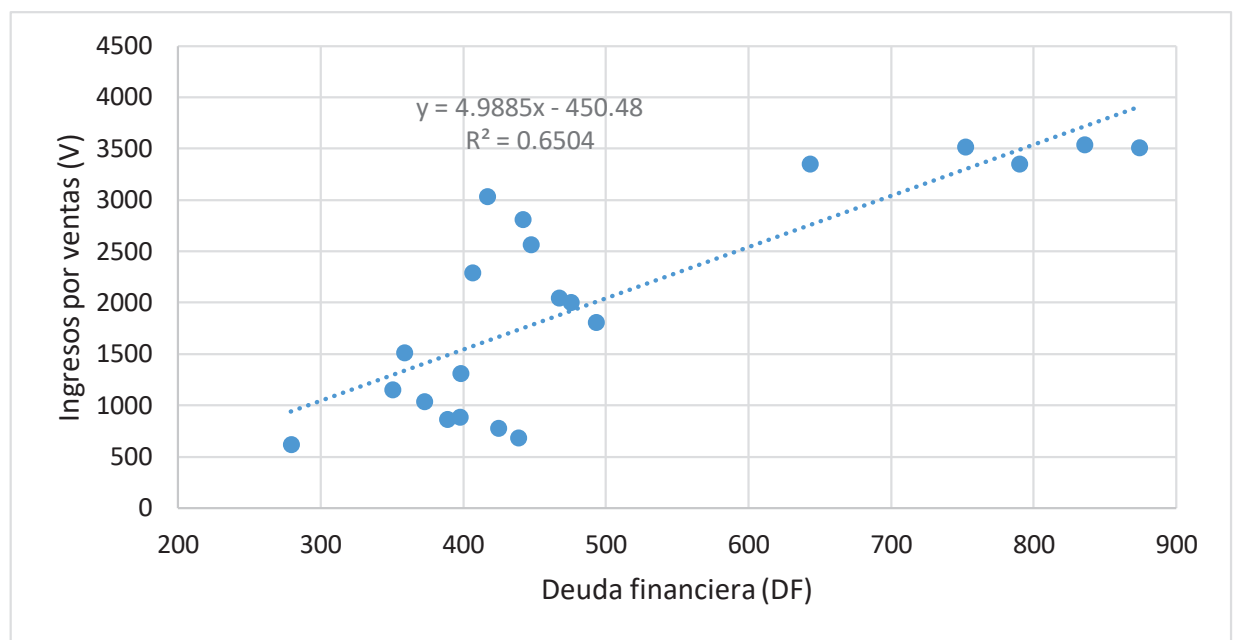

Figura 4. Dispersión de la deuda financiera y los ingresos por ventas: empresa Leche Gloria S.A.

Fuente. Elaboración

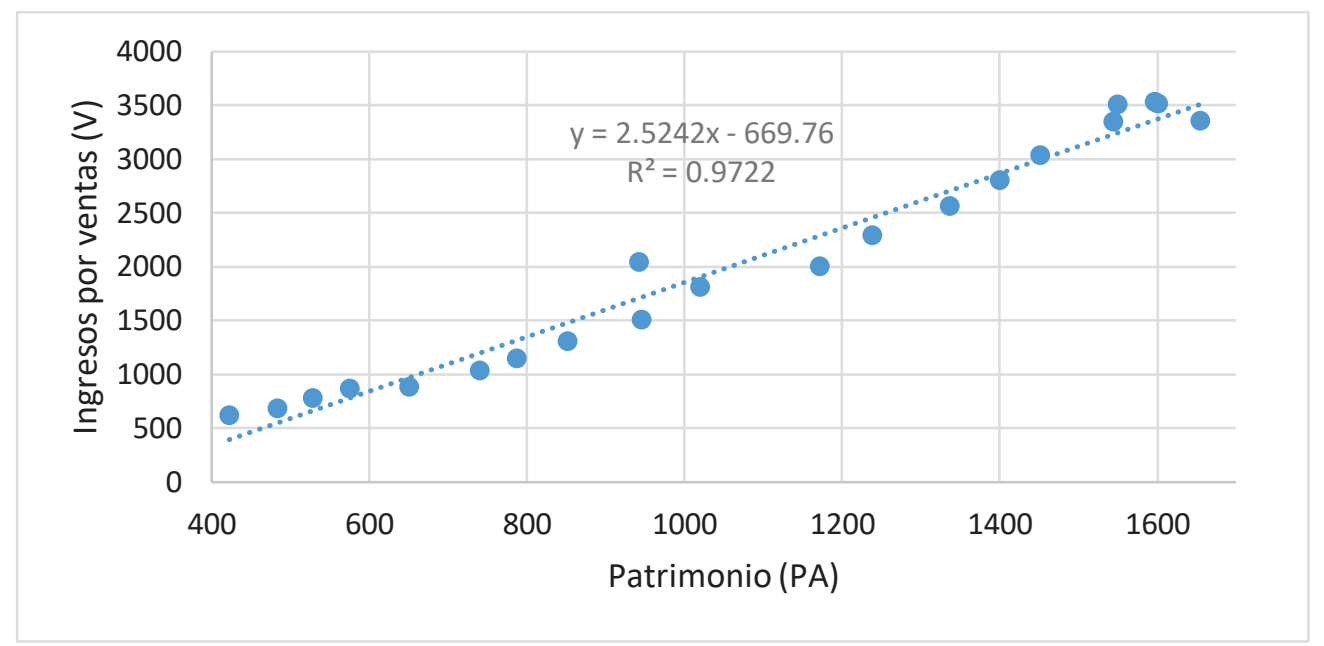

Figura 5. Dispersión de patrimonio y los ingresos por ventas: empresa Leche Gloria S.A.

Fuente. Elaboración propia con datos de la tabla 1 
bondad de ajuste entre las variables independientes y la variable dependiente.

\section{DISCUSIÓN}

Una revisión bibliográfica de la literatura sobre modelos econométricos que relacionen el financiamiento y los ingresos por ventas de una empresa en el Perú ha puesto de manifiesto la carencia de trabajos en el campo de estudio de las finanzas, lo que remite a promover investigaciones futuras que aborden este tema y establezcan propuestas de modelos econométricos eficientes que permitan a la gerencia tomar decisiones adecuadas en su gestión financiera. Se coincide con Ross, Westerfield y Jaffe (2012) que manifiestan "como es evidente, el financiamiento externo necesario y el crecimiento están relacionados. Si no intervienen otros factores, cuanto más alta sea la tasa de crecimiento de ventas o activos, mayor será la necesidad de financiamiento externo" (p. 68). El financiamiento externo está conformado por deuda financiera, que es igual a la suma de deuda financiera de corto plazo y la deuda financiera de largo plazo, que en el caso de la empresa Leche Gloria S.A. creció a una tasa promedio anual compuesto de 5,87\%. Asimismo, se concuerda con Van Horne y Wachowicz (2010) que manifiestan que:

"Con frecuencia, el financiamiento inicial proviene de los fundadores y sus familiares y amigos. En el caso de algunas compañías, esto es suficiente para la puesta en marcha, y si retienen utilidades en el futuro ya no necesitarán financiamiento de capital accionario externo. Para otras son necesarias las infusiones de capital accionario externo adicional" (p.579).

En el caso de la empresa Leche Gloria S.A. ha venido reteniendo y reinvirtiendo utilidades para financiar y coadyuvar al crecimiento de sus ingresos por ventas durante el periodo estudiado a un promedio compuesto anual de $6,72 \%$. Igualmente, en el presente estudio se corroboran la afirmación de Bravo (2017): "Es tal vez importante tomar decisiones de financiamiento para facilitar el crecimiento de la empresa a través de deuda o emisión de acciones" (párr. 1); justamente es lo que hizo la empresa Leche Gloria S.A. como se puede apreciar en la tabla 1, que aumentó su deuda mediante préstamos bancarios y emisión de bonos en $5,87 \%$, si bien es cierto no aumenta el capital social con nueva emisión de acciones, sí aumentó la reinversión de utilidades netas y, por lo tanto, aumentó el financiamiento con patrimonio.

Finalmente, se confirma que el financiamiento con deuda financiera y patrimonio influyeron positiva y significativamente en los ingresos por ventas de la empresa Leche
Gloria S.A. durante el periodo 1998-2018, esto fue demostrado de manera individual mediante el estadístico $t$ de Student calculado, $\mathrm{t}_{\mathrm{c}-\mathrm{PA}}=17,20 \mathrm{y} \mathrm{t}_{\mathrm{c}-\mathrm{DF}}=2,64$ mayores al $\mathrm{t}$ de tabla, $\mathrm{t}_{\mathrm{t}}=2,12$; y de manera conjunta el modelo econométrico queda demostrado mediante el estadístico F. El F calculado es $\mathrm{F}_{\mathrm{c}}=440,00$ mayor al $\mathrm{F}$ de tabla $\mathrm{F}_{\mathrm{t}}=3,55$.

\section{REFERENCIAS}

Bolsa de Valores de Lima (2008). Estado financiero anual individual del año 2008. Gloria S.A. In forme auditado. Recuperado de: https://www.bvl. com.pe/jsp/ShowEEFF_new.jsp?Ano=2008\&Trimestre $=\mathrm{A} \& \mathrm{Rpj}=006166 \&$ RazoSoci $=$ GLORIA $\% 20$ S.A.\&TipoEEFF=I\&Tipo1=A\&Tipo2=I\&Dsc_Correlativo $=0000 \&$ Secuencia $=1$

Bravo, S. (25 de septiembre de 2017). La importancia de las finanzas en la gestión empresarial [mensaje en blog]. Recuperado de https://sergiobravoorellana. com/blog/la-importancia-de-las-finanzas-en-la-gestion-empresarial

Forbes México. (7 de enero de 2016). Los siete pilares para el crecimiento de una empresa. Recuperado de: https:// www.forbes.com.mx/los-7-pilares-para-el-crecimiento-de-una-empresa

Levy, N. (2017). Financiamiento, Financiarización y problemas del desarrollo. Cuadernos de Economía, 38(76), 207-229. Recuperado de: http://www.scielo.org.co/ scielo.php?pid=S0121-47722019000100207\&script $=$ sci_abstract\&tlng=es

Madura, J. (2008). Mercados e instituciones financieras. México: Cengage Learning.

Moyer, R. Ch., McGuigan, J. R. y Kretlow, W. J. (2003). Administración Financiera Contemporánea. México D.F.: Thompson.

Ross, S., Westerfield, R. y Jaffe J. (2012). Finanzas Corporativas. México D.F.: McGraw-Hill.

Van Horne J. y Wachowicz J. (2010). Fundamentos de Administración Financiera. México D.F.: Pearson.

Vásquez, J. y Zamudio, E. (2013). Bóvedas de bancos y el crecimiento económico en el Perú. Revista Moneda, (155), 42-46. Recuperado de https://www.bcrp.gob.pe/ docs/Publicaciones/Revista-Moneda/moneda-155/ moneda-155-08.pdf

Weston, J.F. y Copeland, T.E. (1992). Finanzas en Administración. México D.F.: McGraw Hill/Interamericano de México. 